\title{
Prevalence and risk factors for deep vein thrombosis in hospitalized high-risk patients at a tertiary care hospital in Thailand
}

\author{
Somchai Wongkhantee ${ }^{\mathrm{a}}$, Vigrom Jennetisin ${ }^{\mathrm{a}}$, Thitima Anukulanantchai ${ }^{\mathrm{b}}$, Kittisak Sawanyawisuth,d \\ ${ }^{a}$ Department of Medicine, ${ }^{b}$ Department of Radiology, Khon Kaen Hospital, ${ }^{c}$ Department of Medicine, \\ Faculty of Medicine, Khon Kaen University, ${ }^{d}$ Research Center in Back, Neck Other Joint Pain and \\ Human Performance (BNOJPH), Khon Kaen University, Khon Kaen 40002, Thailand
}

\begin{abstract}
Background: Deep vein thrombosis (DVT) is a silent killer in hospitalized patients. Its prevalence in Thai population may be different from Western countries.

Objective: To evaluate recognized risk factors for DVT in high-risk Thai patients according to the American College of Chest Physicians (ACCP).

Methods: Hospitalized patients were prospectively enrolled at the Department of Medicine, Khon Kean Hospital. The study period was January 2010 to March 2011. Inclusion criteria were patients who were admitted with either (1) heart or respiratory failure or (2) one of the following conditions: cancer, sepsis, acute stroke, or inflammatory bowel disease. The admission length was at least four days. Patients were excluded if the hospitalization was because of venous thromboembolism or if there was a requirement for heparin therapy.

Results: One hundred patients met the study criteria during the study. Of those, 5 patients (5\%) had evidence of DVT by ultrasonography. None of these patients had pulmonary embolisms by computed tomographic angiography. In the multiple logistic regression analysis, only acute stroke was significantly associated with DVT. The adjusted odds ratio (OR) was 19.72; (95\% confidence interval (CI) 1.37, 284.37). DVT was the only significant factor associated with death during admission with adjusted OR of 30.76 (95\% CI: 2.02, 469.03).

Conclusions: The prevalence of DVT in hospitalized high-risk patients by ACCP criteria was low. Admission because of acute stroke may increase the risk of DVT and having DVT may result in high mortality.
\end{abstract}

Keywords: CT angiogram, deep venous thrombosis, high risk, pulmonary embolism, Thai

Venous thromboembolism (VTE) is a silent killer and can result in sudden death [1]. The prevalence of VTE can vary from 5\%-30\% and may be different by ethnicity [2-5]. The American College of Chest Physicians [6] recommended that hospitalized patients who are acutely ill and admitted because of heart failure or respiratory failure are at a high risk of deep venous thrombosis (DVT). In addition, advanced cancer, a previous history of VTE, sepsis, acute stroke, or inflammatory bowel disease also increase risk for DVT [6]. Primary prevention with heparin is beneficial.

The prevalence of DVT in the general population is significantly lower in Asian Americans than white

Correspondence to: Kittisak Sawanyawisuth, MD, PhD, Department of Medicine, Faculty of Medicine, Khon Kaen University, Khon Kaen 40002, Thailand. E-mail: kittisak@ kku.ac.th
Americans [7]. The prevalence of DVT among Asians and Pacific Islanders living in California was 6 in 100,000 and 23 in 100,000 in white people living in California [7]. Data regarding prevalence and risk factors of DVT in Thais are still limited. This study aimed to evaluate risk factors for DVT by ACCP criteria in hospitalized Thai patients at a tertiary care hospital.

\section{Methods}

The study protocol was approved by the ethics committee for human research, Khon Kaen Hospital (58/01/2553). All eligible patients provided signed written informed consent before their participation in the study. Hospitalized patients were enrolled prospectively at the Department of Medicine, Khon Kean Hospital. The study period was January 2010 to March 2011. The inclusion criteria were (1) heart or respiratory failure or (2) one of the 
following conditions; cancer, sepsis, acute stroke, or inflammatory bowel disease. The admission length must be at least four days. Patients were excluded if initial hospitalization was as a result of VTE or requirements for heparin therapy. Definitions of all mentioned variables are described elsewhere [6].

Detection of DVT was performed by limited compression ultrasound and Doppler ultrasound at common femoral veins and popliteal veins by one radiologist (TA). The sensitivity and specificity of these ultrasound techniques are 65\% and 96\% [8]. The investigations were performed on day four of admission in all patients.

Detection of pulmonary embolism was performed within 14 days of admission in all patients. The diagnosis of pulmonary embolism was made by the Modified Wells Pretest Probability Scoring of Pulmonary Embolism [9] and computed tomographic (CT) angiography.

Sample size calculation based on previous studies [3-5], the incidence of DVT was 5\% in hospitalized patients with a deviation of $5 \%$ and two-sided confidence level of 95\% and therefore a required study population of 73.

Eligible patients were divided into two groups; having DVT or no DVT on evidence of ultrasonography. Baseline and clinical characteristics of patients of both groups were compared using descriptive statistics. Wilcoxon rank-sum and Fisher's Exact tests were applied to compare the differences in numbers and proportions between the two group.

Univariate logistic regression analyses were applied to calculate the crude odds ratios of individual variables for having DVT. All clinically significant variables or $P<0.20$ by unvaried analyses were included in subsequent multivariate logistic regression analyses. Analytical results are presented as crude odds ratios (OR), adjusted OR, and 95\% confidence intervals (CI). All factors in multivariate analysis for having DVT were included in the multivariate logistic analysis for death. All data analyses were performed with STATA software (College station, Texas, USA).

\section{Results}

There were 100 patients who met the study criteria during the study period. Of those, 5 patients had evidence of DVT by ultrasonography. None of the patients had pulmonary embolisms by CT angiogram. All clinical variables were comparable between those who had DVT and those who did not have DVT (Table 1). Among those who had DVT, there was a higher proportion of patients with a body mass index equal to or more than $30 \mathrm{~kg} / \mathrm{m}^{2}$ compared with those who did not have DVT (1/5 vs. 1/95, $P=0.098)$. The patients with an acute stroke had a significantly higher number of DVTs (3/5 vs. 16/95, $P=0.046$ ) than the other patients in the study. Four patients in the DVT group died during admission, while 12 patients in the no DVT group died $(P=0.002)$.

Table 1. Clinical factors of patients categorized the presence of deep venous thrombosis (DVT)

\begin{tabular}{llll}
\hline Variables & $\begin{array}{l}\text { NoDVT } \\
(\mathbf{n = 9 5 )}\end{array}$ & $\begin{array}{l}\text { DVT } \\
(\mathbf{n = 5})\end{array}$ & $\boldsymbol{P}$ \\
\hline Age (range years) & $59(17-88)$ & $70(42-83)$ & 0.18 \\
Male & 67 & 3 & 0.64 \\
Underlying disease & & & 0.11 \\
$\quad$ Cancer & 10 & 2 & 0.098 \\
BMI $\geq 30$ kg/m ${ }^{2}$ & 1 & 1 & 0.23 \\
Respiratory failure & 4 & 1 & 0.999 \\
$\quad$ Myeloproliferative disease & 1 & 0 & 0.999 \\
Congestive heart failure & 10 & 0 & 0.999 \\
Admission cause & & & 0.999 \\
Congestive heart failure & 16 & 0 & 0.38 \\
$\quad$ Respiratory failure & 30 & 1 & 0.33 \\
Active cancer disease & 8 & 1 & 0.046 \\
Sepsis & 25 & 0 & 0.09 \\
$\quad$ Acute stroke & 16 & 3 & 0.002 \\
D-dimer, $\mu$ gg/ml (range) & $0.6(0.1-20)$ & $2(0.5-8.6)$ & 4 \\
Death & 12 & 4 & \\
\hline
\end{tabular}

Data presented as median (range) or numbers of patients 
In the multiple logistic regression analysis, only acute stroke was significantly associated with having DVT. The adjusted OR (95\% CI) was 19.72 (95\% CI: 1.37, 284.37) as shown in Table 2. DVT was the only significant factor associated with death during admission with adjusted OR of 30.76 (95\% CI: 2.02, 469.03) as shown in Table 3.

\section{Discussion}

The prevalence rate of DVT in hospitalized medically ill patients found at our Thai tertiary care hospital was 5\%. Reports from the Western countries show that the incidence rate of DVT varied from $4.96 \%$ to $14.9 \%$ [3-5]. As previously reported, the incidence of DVT in Asian Americans may be lower than in white Americans. Different DVT incidences among races may be the result of genetic factors. Factor V Leiden is found in $0.5 \%$ of Asian populations, but up to $5 \%$ in white people. The activity of Protein C may also be less active in Asians than in white people [10].

In the present study, acute stroke is shown to significantly increase risk of DVT in medically ill patients. The risk of DVT in acute stroke patients is increased by 19 times compared with other risk factors (Table 2). A report from China found that the prevalence of DVT in stroke patients was 21.7\% [11].
The present study found the prevalence of DVT in acute stroke somewhat higher than the study from China at 3/5 (Table 1). Immobilization may be an important risk factor of DVT in acute stroke patients.

Even though prevalence of DVT was low in this study, it was an independent factor for death during admission. Having DVT increases risk for mortality by 30 times after adjustment for all clinical variables in Table 3. A D-dimer study may be useful in the diagnosis of DVT or pulmonary embolism, but it did not predict mortality in these high risk patients. Our data suggest that Thai patients with a high risk for DVT by ACCP criteria may have an overall low risk of developing DVT. If DVT does occur in these patients, the risk of death is 30 times greater.

A strength of this study is that all patients with a high risk for DVT were studied specifically for its occurrence and for pulmonary embolism. However, the study population was is small and may not represent the entire Thai population from other medical and surgical categories.

In conclusion, the prevalence of DVT in highrisk hospitalized patients by ACCP criteria was somewhat lower than found previously in Western countries. Admission because of acute stroke may increase risk of DVT and having DVT may result in high mortality.

Table 2. Multivariate logistic regression analysis for having deep venous thrombosis

\begin{tabular}{lll}
\hline Variables & $\begin{array}{l}\text { Univariate odds ratio } \\
\text { (95\% confidence interval) }\end{array}$ & $\begin{array}{l}\text { Adjusted odds ratio } \\
\text { (95\% confidence interval) }\end{array}$ \\
\hline Age, year & $1.04(0.98,1.12)$ & $1.08(0.98,1.19)$ \\
Body mass index $\geq 30 \mathrm{~kg} / \mathrm{m}^{2}$ & $23.50(1.23,447.59)$ & $24.97(0.58,1067.15)$ \\
Acute stroke & $7.41(1.14,47.96)$ & $19.72(1.37,284.37)$ \\
Active cancer disease & $2.72(0.27,27.33)$ & $18.98(0.82,441.34)$ \\
D-dimer & $1.11(0.91,1.36)$ & $1.07(0.86,1.32)$ \\
\hline
\end{tabular}

Bold indicates significant factors by multivariate logistic regression analysis

Table 3. Multivariate logistic regression analysis for death in patients admitted with a high risk for deep venous thrombosis

\begin{tabular}{lll}
\hline Variables & $\begin{array}{l}\text { Univariate odds ratio } \\
\text { (95\% confidence interval) }\end{array}$ & $\begin{array}{l}\text { Adjusted odds ratio } \\
\text { (95\% confidence interval) }\end{array}$ \\
\hline Deep venous thrombosis & $27.67(2.85,268.70)$ & $30.76(2.02,469.03)$ \\
Age, year & $1.02(0.99,1.06)$ & $1.02(0.98,1.05)$ \\
Body mass index $\geq 30 \mathrm{~kg} / \mathrm{m}^{2}$ & $5.53(0.33,93.37)$ & $1.18(0.02,57.21)$ \\
Acute stroke & $1.53(0.43,5.41)$ & $0.73(0.13,4.20)$ \\
Active cancer disease & $0.63(0.07,5.44)$ & $0.30(0.01,6.20)$ \\
D-dimer & $1.06(0.90,1.24)$ & $1.04(0.86,1.26)$ \\
\hline
\end{tabular}

Bold indicates significant factors by multivariate logistic regression analysis 


\section{Acknowledgments}

This study was supported by TRF grants from Senior Research Scholar Grant, Thailand Research Fund grant number RTA5580004 and the Higher Education Research Promotion and National Research University Project of Thailand, Office of the Higher Education Commission, Thailand through the Health Cluster (SHeP-GMS), Khon Kaen University. The authors would like to thank Prof. James A. Will (University of Wisconsin, USA) for his kind review of the manuscript. This study also received partial financial support from Sanofi-aventis Thailand Ltd.

The authors have no conflicts of interest to declare.

\section{References}

1. Baglin TP, White K, Charles A. Fatal pulmonary embolism in hospitalized medical patients. J Clin Pathol. 1997; 50:609-10.

2. Cade JF. High risk of the critically ill for venous thromboembolism. Crit Care Med. 1982; 10:448-50.

3. Cohen AT, Davidson BL, Gallus AS, Lassen MR, Prins MH, Tomkowski W, et al. Efficacy and safety of fondaparinux for the prevention of venous thromboembolism in older acute medical patients: randomized placebo controlled trial. BMJ. 2006; 332: 325-9.

4. Leizorovicz A, Cohen AT, Turpie AG, Olsson CG, Vaitkus PT, Goldhaber SZ, et al. Randomized, placebocontrolled trial of dalteparin for the prevention of venous thromboembolism in acutely ill medical patients. Circulation. 2004; 110:874-9.
5. Samama MM, Cohen AT, Darmon JY, Desjardins L, Eldor A, Janbon C, et al. A comparison of enoxaparin with placebo for the prevention of venous thromboembolism in acutely ill medical patients. Prophylaxis in Medical Patients with Enoxaparin Study Group. N Engl J Med. 1999; 341:793-800.

6. Bates SM, Greer IA, Pabinger I, Sofaer S, Hirsh J; American College of Chest Physicians. Venous thromboembolism, thrombophilia, antithrombotic therapy, and pregnancy: American College of Chest Physicians Evidence-Based Clinical Practice Guidelines (8th Edition). Chest. 2008; 133:844S-86S.

7. White RH, Zhou H, Romano PS. Incidence of idiopathic deep venous thrombosis and secondary thromboembolism among ethnic groups in California. Ann Intern Med. 1998; 128:737-40

8. Kearon C, Julian JA, Newman TE, Ginsberg JS. Noninvasive diagnosis of deep vein thrombosis. McMaster Diagnostic Imaging Practice Guidelines Initiative. Ann Intern Med. 1998; 128:663-77.

9. Wells PS, Brill-Edwards P, Stevens P, Panju A, Patel A, Douketis J, et al. A novel and rapid whole-blood assay for D-dimer in patients with clinically suspected deep vein thrombosis. Circulation. 1995; 1:2184-7.

10. White RH. The epidemiology of venous thromboembolism. Circulation. 2003; 107:I4-8.

11. Sun KK, Wang C, Pang BS, Yang YH, He W, Chen TF, et al. [Study on the risk factors of deep venous thrombosis in acute hospitalized stroke patients]. Zhonghua Liu Xing Bing Xue Za Zhi. 2004; 25: 10019-23. [Article in Chinese] 